\title{
Evaluación de la eficiencia de las compañías aéreas brasileñas a través de un modelo híbrido de análisis envolvente de datos (DEA) y programación lineal multiobjetivo
}

\section{Brazilian Airlines efficiency evaluation using a data envelopment analysis (DEA) and multiobjective linear programming hybrid model}

\author{
Juliana Quintanilha da Silveira $^{1} \quad$ Joao Carlos Correia Baptista Soares de Mello $^{1}$ \\ Lidia Angulo Meza ${ }^{1}$
}

Recibido 7 de diciembre de 2010, aceptado 13 de agosto de 2012

Received: December 7, 2010 Accepted: August 13, 2012

\begin{abstract}
RESUMEN
El aumento creciente de la competitividad del sector de transporte aéreo en los últimos años provocó un cambio en la dinámica del mercado, lo que ha llevado a las compañías aéreas a buscar nuevas estrategias para garantizar sus posiciones en el mercado. Este artículo tiene por objetivo hacer un análisis del desempeño de las compañías aéreas brasileñas, con relación a su gestión operacional después de la consolidación de los cambios resultantes de la liberalización del sector en el año 2005. La eficiencia de las empresas aéreas se calcula a través de la comparación de los resultados obtenidos con el modelo del Análisis Envolvente de Datos (DEA) clásico con los índices de eficiencia calculados con base en el modelo multiobjetivo MCDEA-BCC, desarrollado para este estudio a partir del modelo MCDEA de Li e Reeves. Además este trabajo incluye la segmentación de las empresas en agrupamientos y la identificación de benchmarks, en la que se indican las compañías con las mejores prácticas gerenciales del sector aéreo.
\end{abstract}

Palabras clave: Transporte aéreo, análisis envolvente de datos, programación lineal multiobjetivo, MCDEA, TRIMAP.

\section{ABSTRACT}

The increase of the air transportation sector competition in recent years has changed this market dynamics, leading airlines to seek new strategies to ensure its position. This article aims to analyze the performance of Brazilian air carriers regarding their operational management, after the consolidation of the changes arising from the deregulation of the sector, in the year 2005. The analysis of the companies' efficiency in the year 2005 is done by comparing the results obtained with Data Envelopment Analysis model and with efficiency index based on the multiobjective model MCDEA-BCC, developed in this study from the Li and Reeves' MCDEA model. This project included a segmentation of the companies in clusters and benchmark, indicating the best managerial practices in the air sector.

Keywords: Air transportation, data envelopment analysis, multiobjective linear programming, MCDEA, TRIMAP.

\section{INTRODUCCIÓN}

El sector de transporte aéreo en el Brasil ha pasado por transformaciones estructurales significativas a lo largo de los últimos años. Desde el inicio de la década de los 90, el sector presenció un proceso de liberalización (deregulamentation), que rompió con la política vigente, instalada desde el final de los

\footnotetext{
1 Pos Grado en Ingeniería de Producción. Universidade Federal Fluminense. Rua Passo da Pátria 156, 22210-240. Niterói, Brasil. E-mail: juliqs@hotmail.com; jcsmello@pq.cnpq.br; lidia_a_meza@pq.cnpq.br
} 
años 60 , en la cual la estructura y la conducta del mercado eran controladas y asociadas a mecanismos de política industrial [1].

El inicio de este proceso surgió a partir de la abolición de los monopolios regionales en 1992. De esta manera, se estimuló la entrada de nuevas operadoras, además de la competencia de precios con la definición de categorías de tarifas. Al final de los años 90, la liberalización tuvo un nuevo impulso [2], se removieron las categorías de tarifas y la exclusividad que todavía existía en la operación de algunas líneas aéreas para las compañías regionales. Estas medidas amplificaron mucho más la competencia entre las compañías. En 2001, hubo una liberación total de precios, una flexibilización de los procedimientos de entrada de nuevas empresas y de los pedidos de nuevas líneas aéreas, de las frecuencias de vuelo y aviones, lo que tuvo como resultado la entrada de la compañía aérea Gol, la primera empresa Low Cost Carrier (LCC), de bajo costo, en enero de 2001.

La competencia establecida entre las empresas de transporte aéreo acarrea la búsqueda por el mejor desempeño, lo que conlleva a que desarrollen nuevas estrategias para garantizar su posición en el mercado.

Este artículo tiene por objetivo evaluar el desempeño de las compañías aéreas brasileras para el transporte aéreo de pasajeros y cargas, en el año de 2005, después de la consolidación de los cambios descritos anteriormente. Con este objetivo, se aplicará el Análisis Envolvente de Datos (Data Envelopment Analysis, DEA) a las compañías que estaban en operación en el año en cuestión y el Modelo de Análisis Envolvente de Datos Multicriterio (Multicriteria Data Envelopment Analysis, MCDEA) para buscar el aumento de la capacidad de distinción del método clásico cuando se evalúan las eficiencias operacionales de las compañías.

Para generar soluciones no dominadas para el modelo multiobjetivo se utilizó la herramienta computacional TRIMAP, desarrollada por Clímaco e Antunes [3], la cual permite una evaluación con relación a la composición del espacio de los pesos de las funciones objetivo, para el caso de tres funciones objetivo. A partir de los resultados obtenidos se determinó, para cada compañía aérea, el índice de eficiencia MCDEA-TRIMAP propuesto por Soares de Mello, Clímaco y Angulo-Meza [4].
Además, se indicarán las compañías con las mejores prácticas de gestión, a través de la segmentación de las compañías en agrupamientos o clusters, los que serán definidos a partir de los multiplicadores atribuidos por cada compañía a sus recursos y productos.

Por otro lado, es importante destacar que los estudios de eficiencia en empresas aéreas son escasos. Sobre compañías brasileñas, se encontraron dos estudios sobre eficiencia operacional [5-6] y otros dos que utilizan DEA para estudiar la estructura de capital de esas empresas [7-8]. Utilizando el modelo de Li e Reeves usado en este artículo, pero utilizando retornos constantes de escala se realizó la evaluación de las empresas aéreas por Silveira, Pereira, Correia, Soares de Mello, Clímaco y Angulo-Meza [9]. Con relación al transporte aéreo, la herramienta DEA es más utilizada para estudiar las eficiencias de los aeropuertos [10-13].

Este trabajo está organizado de la siguiente manera: en la sección siguiente, El Análisis Envolvente de Datos y el Modelo de Li e Reeves, se encuentra una revisión del modelo DEA clásico y del modelo de Li e Reeves. A continuación, en la sección TRIMAP y el Modelo MCDEA se discute el uso del software TRIMAP en conjunto con el modelo MCDEA. Posteriormente, se describe el Índice MCDEATRIMAP y a continuación, en la sección Cálculo del Conjunto de Multiplicadores, se describe el procedimiento para el cálculo de los multiplicadores de las variables de cada compañía aérea analizada en este trabajo. La caracterización y el modelamiento del problema están en la sección siguiente, Evaluación de las Compañías Aéreas Brasileñas. A continuación se presentan los resultados de la aplicación del modelo MCDEA, junto con el cálculo del Índice de Eficiencia y a la segmentación en clusters. Finalmente, en Conclusiones se presentan conclusiones de este trabajo y propuestas para trabajos posteriores.

\section{EL ANÁLISIS ENVOLVENTE DE DATOS Y EL MODELO DE LI E REEVES}

El Análisis Envolvente de Datos es una metodología basada en programación matemática, que tiene por objetivo medir la eficiencia de un conjunto de unidades productivas (unidades tomadoras de decisión), llamadas DMUs (Decision Making Units), 
que consumen múltiples inputs (insumos, recursos) para producir múltiples outputs (productos).

Existen dos modelos clásicos en DEA: el CCR (también conocido como CRS o Constant Return to Scale) propuesto por A. Charnes, W.W. Cooper y E. Rhodes [14] y el BCC (también conocido como VRS o Variable Return to Scale) propuesto por R.D. Banker, A. Charnes y W.W. Cooper [15]. En el modelo CCR cualquier variación en los inputs produce una variación proporcional en los outputs, considerando retornos constantes de escala. Por el contrario, el modelo BCC no asume proporcionalidad entre inputs y outputs, lo que permite retornos variables de escala.

Para el cálculo de la eficiencia es posible utilizar la orientación a inputs que tiene por objetivo producir la misma cantidad de productos minimizando la utilización de recursos. Por otro lado, la orientación a outputs tiene por objetivo maximizar la producción manteniendo constante la cantidad de recursos consumidos.

En DEA, cada DMU escoge, a través del problema de programación matemática, su propio conjunto de multiplicadores, de forma que la eficiencia sea la mejor posible en comparación con las demás. Debido a esto, es posible que un gran número de DMUs se ubique en la frontera eficiente, lo que revela una estructura benevolente de este método y lo que reduce su capacidad de distinción o discriminación. Según F.R. Leta, J.C.C.B. Soares de Mello, E.G. Gomes y L. Angulo-Meza [16], y como ya había sido mencionado previamente por T.R. Sexton, R.H. Silkman, A.J. Logan [17], Golany y Roll [18], entre otros, mediante determinación empírica, el empate y gran cantidad de las DMUs que son $100 \%$ eficientes ocurre principalmente cuando el número de DMUs no es muy grande cuando se le compara con el número total de inputs e outputs del análisis.

En los últimos años se han desarrollado diferentes modelos que tienen por objetivo la mejora de la distinción o discriminación en DEA [19]. Entre ellos se puede destacar el modelo MCDEA, de Li e Reeves [20], que utiliza la Programación Lineal Multiobjetivo para resolver los problemas de discriminación de las DMUs y promover una mejor distribución de los multiplicadores de las variables. El modelo MCDEA proporciona soluciones no dominadas, que tienen en cuenta todas las funciones objetivo y que no están limitadas por las soluciones obtenidas a partir de la optimización individual de cada función objetivo.

De esta forma, el modelo MCDEA de Li e Reeves [20] incluye dos funciones objetivo adicionales para restringir la libertad de los pesos cuando se utiliza el modelo DEA-CCR propuesto por A. Charnes, W.W. Cooper y E. Rhodes [14]. Estas dos funciones objetivo representan nuevos criterios para medir la eficiencia y estos tienden a restringir más la eficiencia, ya que permiten menor flexibilidad en la optimización.

El modelo MCDEA propone utilizar una holgura o desviación $d_{o}$ como medida de ineficiencia, en vez de la eficiencia $h_{o}$, siendo que una es complemento de la otra. Así, en este modelo la DMU evaluada $o$ es eficiente si y solamente si $d=0$. Entonces, se puede decir que esta formulación minimiza la ineficiencia de la DMU en evaluación, con la restricción de que la suma ponderada de los outputs sea menor o igual a la suma ponderada de los inputs de cada DMU. Las dos funciones objetivo adicionales: la minimización de la suma de las holguras desviaciones de cada restricción (que se puede considerar como "benevolencia generalizada") y la minimización de la máxima desviación (que se puede considerar como “equidad"). De acuerdo con Li y Reeves [20], cada una de las tres funciones objetivo es independiente con relación a las demás, en que no hay una orden de prioridad entre los criterios de eficiencia.

En este estudio se formuló el modelo MCDEA de manera análoga para el modelo DEA-BCC a partir de los conceptos discutidos anteriormente. Así, la formulación (1) muestra el modelo MCDEA-BCC.

Min $d_{0}$

$\operatorname{Min} \sum_{j=1}^{n} d_{j}$

Min Max $d_{j}$

sujeto a

$\sum_{i=1}^{r} v_{i} x_{i o}=1$

$\sum_{i=1}^{r} v_{i} x_{i k}-\sum_{j=1}^{s} u_{j} y_{j k}+d_{0}+u_{*} \leq 0, \quad k=1, . ., n$

$u_{j}, v_{i} \geq 0, \forall j, i$

$u_{*} \in \Re$ 
En esta formulación (1) $v_{i}$ y $u_{r}$ son los multiplicadores de inputs $i, i=1, \ldots, m$, y outputs $r, r=1, \ldots, s$, respectivamente; $x_{i j}$ y $y_{r j}$ son los inputs $i$ y outputs $r$ de la DMU $j, j=1, \ldots, n ; u_{*}$ es la variable de escala; $d_{j}$ es la holgura de la DMU $j, j=1, \ldots, n$, y $d_{o}$ es la holgura de la DMU $O$.

Cabe resaltar que debido a la formulación del BCC clásico, el modelo MCDEA-BCC puede generar holguras mayores que uno, lo que significa eficiencias negativas. Según J.C.C.B. Soares de Mello, M.P.E. Lins y E.G. Gomes [21], este fenómeno ocurre especialmente cuando una DMU tiene retornos crecientes de escala.

Para evaluar los resultados, una DMU es eficiente minisuma si, y solamente si, el valor de la función objetivo correspondiente a la solución que minimiza la segunda función objetivo del modelo es cero. Análogamente, se considera que una DMU es eficiente minimax si y solamente si el valor correspondiente a la solución que minimiza la tercera función objetivo del modelo MCDEA es cero.

Cuando una DMU es minimax o minisuma eficiente también debe ser DEA eficiente, en el sentido clásico, ya que por definición las eficiencias minimax o minisuma requieren $d_{o}=0$.

Sin embargo, en casos particulares, el objetivo minimax puede restringir poco las combinaciones de los multiplicadores que optimizan la función objetivo clásica. Habitualmente, eso ocurre cuando la DMU con peor evaluación tiene puntos fuertes y débiles semejantes a la DMU en evaluación, esto significa que tiene multiplicadores semejantes.

\section{TRIMAP Y EL MODELO MCDEA}

El TRIMAP [22] es una herramienta de apoyo a la decisión que tiene por objetivo apoyar a los agentes de decisión en la búsqueda de soluciones eficientes para problema de programación lineal tricriterio. Este método combina una reducción de la región factible, con la reducción de los pesos de las funciones objetivo, lo que permite que el agente decidor especifique límites inferiores para los valores de la función objetivo y/o imponga restricciones en el espacio de los pesos [23].
Inicialmente, en el método TRIMAP se calculan las soluciones eficientes que optimizan cada función objetivo y la solución eficiente que minimiza una distancia ponderada de Tchebycheff a la solución ideal. El hecho de trabajar apenas con tres funciones objetivo, muchas veces representa una limitación del método. Sin embargo, el TRIMAP permite utilizar representaciones gráficas bastante útiles, como la representación del espacio de los pesos de las funciones objetivo, que es de gran interés para el modelo MCDEA. Como el modelo de este artículo tiene exactamente tres funciones objetivo, la limitación previamente mencionada no existe.

El gráfico proporcionado por el TRIMAP muestra el espacio de los pesos descompuesto en regiones de indiferencia (tal como será visto en la Figura 1), que son regiones en que los pesos de las funciones objetivo pueden variar sin alterar los valores encontrados para las funciones objetivo. Las regiones de indiferencia corresponden a las soluciones básicas no dominadas obtenidas hasta el momento. Además, el gráfico puede mostrar restricciones directas a los pesos y a los valores factibles de las funciones objetivo. Un ejemplo de este gráfico será visto en la sección de resultados.

Además, el TRIMAP ofrece un resumen de los resultados numéricos obtenidos y proporciona para cada solución básica no dominada, el valor de las variables básicas, de las funciones objetivo, el porcentual de área ocupada por la región de indiferencia, entre otras informaciones.

Teniendo en consideración las características del modelo MCDEA, el TRIMAP se muestra como una herramienta altamente apropiada para su estudio, pues calcula todas las soluciones óptimas de la función objetivo del modelo DEA clásico e identifica las soluciones no dominadas. J.C.C.B. Soares de Mello, J.C.N. Clímaco y L. Angulo-Meza [4] destacan la importancia de este resultado, ya que el conocimiento de la existencia de multiplicadores alternativos y la identificación de cuáles corresponden a soluciones básicas promueve la realización de análisis más profundos. En este estudio, el cálculo de los multiplicadores atribuidos a cada variable de la DMU, detallado en el tópico 5, se utilizará para la segmentación del mercado de las compañías aéreas brasileñas. 


\section{ÍNDICE MCDEA-TRIMAP}

Propuesto por J.C.C.B. Soares de Mello, J.C.N. Clímaco y L. Angulo-Meza [4], el Índice de Eficiencia MCDEA-TRIMAP considera las propiedades resultantes del uso del TRIMAP para el modelo MCDEA.

La elección de los multiplicadores de cada DMU depende de la atribución de pesos a cada función objetivo, los que pueden variar dentro de una región de indiferencia. Para cada región de indiferencia habrá soluciones diferentes, lo que dificulta una ordenación única. Además no es fácil para un agente decidor atribuir pesos, pues las funciones objetivo minimax y minisuma, a pesar de su clara interpretación matemática, no son triviales de interpretar por el agente decidor, en un contexto de evaluación en DEA.

Para el cálculo de la eficiencia en el modelo MCDEA, debe ser considerada toda la infinidad de combinaciones posibles de los pesos de las funciones objetivo. Además, existe una variación continua de los valores asumidos por la función objetivo clásica, en el espacio de los pesos.

Para definir una forma de ordenación, el Índice MCEA integra la función objetivo clásica cuando se integra la suma ponderada de las tres funciones objetivo. Esa integración debe ser realizada en todo el espacio de los pesos posibles y la división de su resultado por el tamaño de ese espacio proporciona el valor medio de la función objetivo clásica en ese espacio. El complemento de ese valor medio representa el Índice de Eficiencia, conforme detallado por la ecuación (2).

$$
\begin{aligned}
& I\left(E f_{\text {MCDEA-TRIMAP }}\right)= \\
& 1-\left(\left(\iint F O 1\left(\lambda_{1}, \lambda_{2}, \lambda_{3}\right) d S\right) / \text { área del } \Delta\right.
\end{aligned}
$$

En esta ecuación FO1 es el valor asumido por la función objetivo 1, la función objetivo DEA clásica, en la región de indiferencia. $\lambda_{1}, \lambda_{2}, \lambda_{3}$ son los pesos de las funciones objetivo 1,2 e 3 , respectivamente; y área del $\Delta$ es el área o espacio de los pesos de las funciones objetivo.

El cálculo de este índice se puede simplificar si se observa que el integrando es continuo por partes en el espacio de los pesos, y que en cada región de continuidad, asume un valor constante. Por lo tanto, basta calcular la suma ponderada de la primera función objetivo, teniendo como ponderadores los porcentajes de área en que cada solución es válida.

Sin embargo, una pequeña modificación se debe hacer en el modelo MCDEA original, para evitar distorsiones en la integración del espacio de los pesos, generadas por la consideración de varias desviaciones de eficiencia en la función objetivo minisuma, a diferencia de lo que ocurre con las otras dos funciones objetivo. Para eliminar las distorsiones, se divide la expresión referente a esa función objetivo (minisuma) por el número total de DMUs en análisis.

\section{CÁLCULO DEL CONJUNTO DE MULTIPLICADORES}

J.C.C.B. Soares de Mello, J.C.N. Clímaco y L. Angulo-Meza [4] proponen un proceso de cálculo del conjunto de multiplicadores de cada DMU a partir del Índice MCDEA obtenido para cada una de ellas. Para esto, se resuelve un PPL originado en el modelo DEA clásico, en el que se obliga a que la eficiencia sea igual al índice calculado por el modelo MCDEA-TRIMAP, conforme descrito en el modelo (3). En este modelo se maximiza el menor de los multiplicadores mientras se mantienen las otras restricciones del modelo DEA clásico.

Min Max $u_{o}$ sujeto a

$\sum_{i=1}^{r} u_{j} y_{j o}=I\left(E f_{M C D E A-T R I M A P}\right)$

$\sum_{i=1}^{r} v_{i} x_{i o}=1$

$\sum_{i=1}^{r} v_{i} x_{i k}-\sum_{j=1}^{s} u_{j} y_{j k}+u_{*} \leq 0, \quad k=1, . ., n$

$u_{j}, v_{i} \geq 0, \forall j, i$

$u_{*} \in \Re$

En (3) $v_{i}$ y $u_{r}$ son los multiplicadores de inputs $i$, $i=1, \ldots, m$, y outputs $r, r=1, \ldots, s$, respectivamente; $x_{i j}$ y $y_{r j}$ son los inputs $i$ y outputs $r$ de la DMU $j$, $j=1, \ldots, n ; \mathrm{u}_{*}$ es la variable de escala; $\mathrm{u}_{o}$ es el multiplicador de la DMU $O$. 
Caso el agente decisor acepte los multiplicadores calculados y siendo diferentes de cero, el problema termina. Caso el agente decidor no acepte la solución, se pueden colocar restricciones a los multiplicadores y recalcular el problema.

\section{EVALUACIÓN DE LAS COMPAÑIIISS AÉREAS BRASILEÑAS}

El periodo seleccionado para la evaluación de las compañías aéreas brasileñas fue el año 2005, cuando ya se habían consolidado los cambios en el mercado de transporte aéreo brasileño, descritos anteriormente. Este período estuvo marcado por la reducción de las barreras geográficas entre compañías regionales, nacionales e internacionales y por la búsqueda de un mejor aprovechamiento de los recursos disponibles. Así la competencia entre las empresas se volvió más dura.

Además, en el año 2005 hubo una paralización y cierre de las operaciones de la VASP, mientras que nuevas empresas iniciaron sus operaciones regulares. Algunas empresas tradicionales en el segmento no regular se transformaron en concesionarias y pasaron a operar también líneas regulares, como por ejemplo la TAF Líneas Aéreas que volvió a operar en este mercado de donde se había retirado en 2002.

Debido a la diferencia de tamaño entre las compañías aéreas, y como no hay garantía sobre la proporcionalidad entre inputs (capacidad de la flota y total de personal) y outputs (pasajeros.km utilizados y toneladas.km utilizados) se escogió el modelo DEA BCC con orientación a input, pues considera retornos variables de escala. De esta forma, se entiende que para largas distancias el aprovechamiento de las aeronaves es más grande. Un resumen de inputs e outputs se encuentra en el Cuadro 1.

Cuadro 1. Resumen de inputs e outputs

\begin{tabular}{|c|c|}
\hline Inputs & $\begin{array}{c}\text { Capacidad de la flota } \\
\text { Total de personal }\end{array}$ \\
\hline Outputs & $\begin{array}{l}\text { Pasajeros.km utilizados } \\
\text { Toneladas.km utilizados }\end{array}$ \\
\hline
\end{tabular}

Se compararon los resultados de la metodología DEA con los del modelo MCDEA-BCC, desarrollado a partir del modelo de Li y Reeves [20], como método de aumento de la capacidad de discriminación, de tal forma que la medida de las eficiencias operacionales de las compañías tuviese mayor precisión. Además, con la finalidad de identificar compañías con el mismo perfil operacional, se definieron clusters, a través del cálculo del vector de multiplicadores de cada una de las compañías. En cada cluster se identificó la compañía más eficiente, o sea, la meta (benchmark) a ser alcanzada por las otras.

El estudio tiene por objetivo evaluar el desempeño de las compañías aéreas con relación a su gestión operacional. Para esto, como uno de los inputs del modelo (recursos utilizados por cada compañía para poner en operación el servicio) se consideró el principal bien capital de las empresas: las aeronaves. Sin embargo, como las aeronaves utilizadas son de modelos diferentes y, por lo tanto, tienen capacidades de transporte diferentes, se utilizó como variable el peso máximo de decolaje de cada compañía, tal como fue propuesto por Correia y Soares de Mello [24]. De hecho, el peso máximo de decolaje engloba el peso del avión y del combustible (lo que no genera ingresos), más el peso de la carga transportada y el peso de los pasajeros (lo que sí genera ingresos).

Asimismo, se necesita personal para operar las aeronaves, ejecutar los servicios de apoyo y administrar la empresa. Con esta consideración, el otro input utilizado fue el total de personal de cada empresa. Es importante destacar que se podría haber utilizado combustible como otro input, sin embargo, la ANAC dejó de divulgar el total de combustible consumido por cada empresa. Además, J.C.C.B. Soares de Mello, L. Angulo-Meza, E.G. Gomes, B.P. Serapião y M.P.E. Lins [6] mostraron que el uso de esa variable distorsiona los resultados e identifica una cantidad muy grande de empresas como eficientes.

Por otro lado, las empresas transportan pasajeros y carga, y reciben por este transporte, así los outputs deben estar vinculados a esas dos variables. También no basta transportar, el transporte aéreo se caracteriza por transportar a largas distancias. Así, se seleccionaron como outputs el número de asientos utilizados por kilómetros y toneladas utilizadas por kilómetros.

Los datos fueron obtenidos en el Anuario Estadístico del Transporte Aéreo divulgado por la Agencia Nacional de Aviación Civil (ANAC). Este estudio consideró los datos disponibles del año 2005 por ser 
los más recientes en la ocasión en que el estudio se inició. Cabe resaltar que los anuarios de la ANAC proporcionan datos referentes al final del año. De esta forma, aunque no se conozca el comportamiento de la flota a lo largo del año, se puede estimar la flota media a partir de los datos en el inicio del año (flota del fin del año anterior) y del fin del año.

\section{RESULTADOS}

El modelo DEA-BCC clásico se aplicó a las 14 DMUs que representan las empresas brasileñas de transporte aéreo regular, con movimiento de carga y pasajeros en el año 2005. Para el cálculo de las eficiencias mostradas en la Tabla 1, se utilizó el software SIAD [25].

Tabla 1. Eficiencia clásica de las compañías aéreas de 2005.

\begin{tabular}{|c|c|}
\hline $\begin{array}{c}\text { Compañías aéreas brasileñas } \\
\text { en 2005 }\end{array}$ & $\begin{array}{c}\text { Eficiencia } \\
\text { (Eff }_{\mathbf{0}} \text { ) }\end{array}$ \\
\hline Abaeté Linhas Aéreas & 1,0000 \\
\hline Absa-Aerolineas Brasileiras & 1,0000 \\
\hline Gol Transportes Aéreos & 1,0000 \\
\hline Tam Linhas Aéreas & 1,0000 \\
\hline Grupo Varig & 1,0000 \\
\hline Meta Mesquita & 0,9267 \\
\hline Puma Air Linhas Aéreas & 0,8297 \\
\hline Passaredo Transportes Aéreos & 0,8178 \\
\hline Taf Linhas Aérea & 0,7020 \\
\hline Trip Linhas Aéreas & 0,5731 \\
\hline Rico Linhas Aéreas & 0,4396 \\
\hline Pantanal Linhas Aéreas & 0,3723 \\
\hline Oceanair & 0,2066 \\
\hline Total Linhas Aéreas & 0,1992 \\
\hline
\end{tabular}

Mostrando la estructura benevolente del método, hubo "empates", con un resultado de cinco DMUs eficientes en el año 2005 (Abaeté Linhas Aéreas, ABSA Aerolineas Brasileiras, Gol Transportes Aéreos, TAM Linhas Aéreas y Grupo Varig). En este caso no hay como distinguir entre las compañías eficientes usando el modelo DEA-BCC clásico.

Para aumentar la discriminación de las compañías aéreas en el 2005, se aplicó el modelo MCDEABCC con el uso del TRIMAP, con el objetivo de evaluar también el espacio de los pesos. Las Figuras 1, 2, 3, 4 y 5 muestran la descomposición del espacio de los pesos para las DMUs que fueron eficientes en el modelo clásico, después del uso del modelo MCDEA-BCC. En estas figuras $\lambda_{1}$ es el peso asociado a la función objetivo 1 , la eficiencia clásica; $\lambda_{2}$ es el peso asociado a la función objetivo 2, minimización de la suma de las desviaciones; y $\lambda_{3}$ es el peso asociado a la función objetivo 3 , minimización de la máxima desviación.

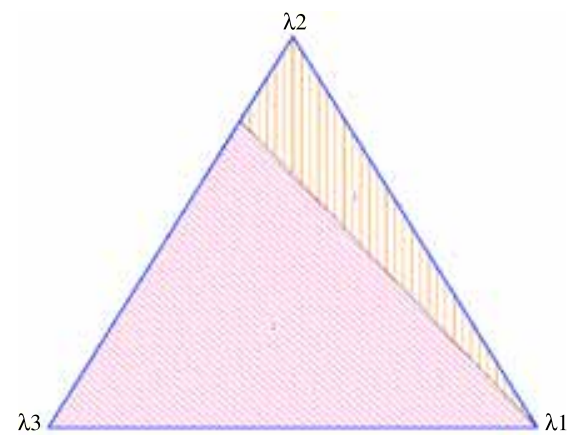

Figura 1. Espacio de los pesos de las funciones objetivo para la DMU Abaeté Linhas Aéreas.

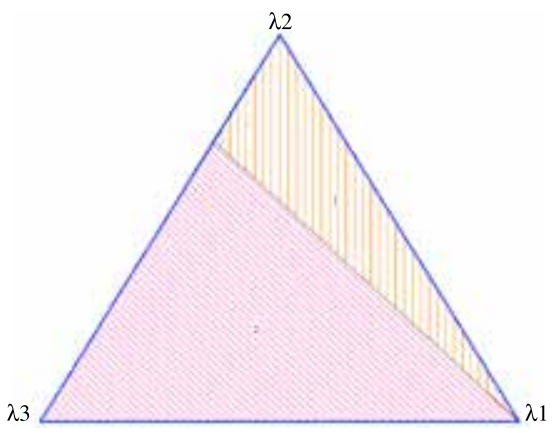

Figura 2. Espacio de los pesos de las funciones objetivo para la DMU ABSA - Aerolíneas Brasileiras.

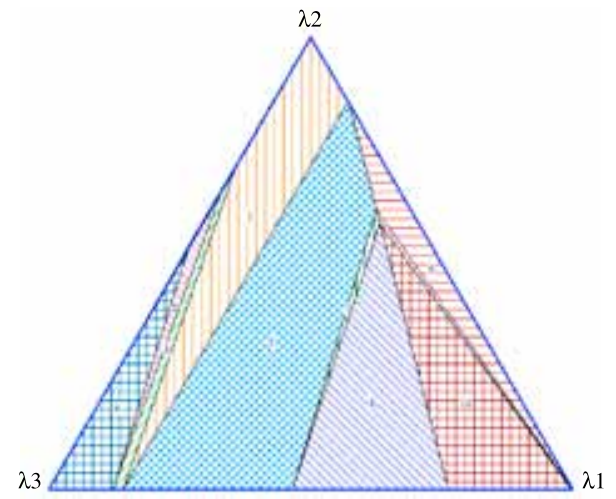

Figura 3. Espacio de los pesos de las funciones objetivo para la DMU Gol Transportes Aéreos. 


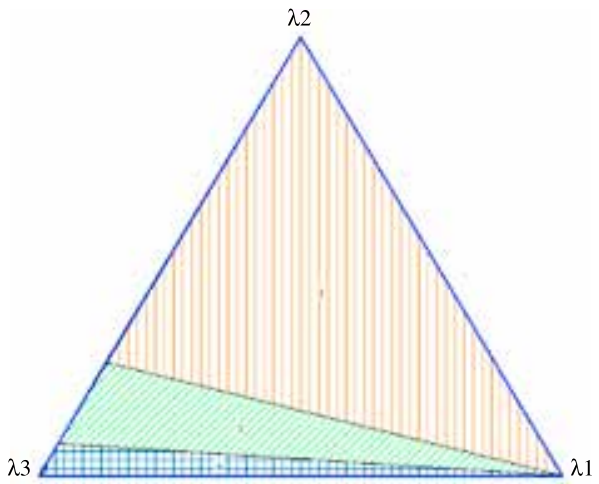

Figura 4. Espacio de los pesos de las funciones objetivo para la DMU TAM.

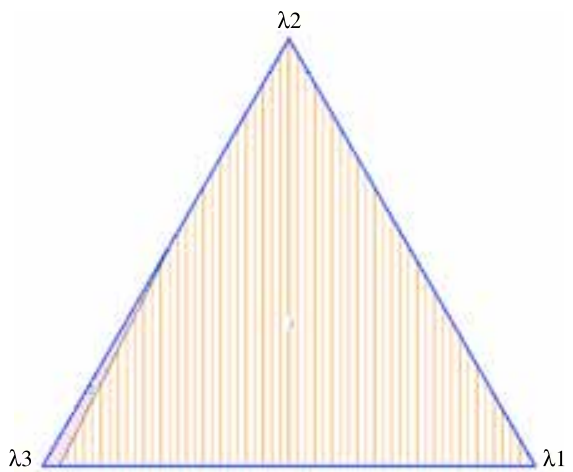

Figura 5. Espacio de los pesos de las funciones objetivo para la DMU Grupo Varig.

Utilizando los procesos de análisis descritos por [26], se verifica que las DMUs Abaeté Linhas Aéreas y ABSA Aerolíneas Brasileiras fueron minisuma y minimax eficientes. Los gráficos muestran que el vértice referente a la función objetivo DEA-BCC clásica (vértice inferior derecho) se encuentra en la misma región de indiferencia que el vértice correspondiente a la función objetivo minisuma (vértice superior), lo que significa decir que una misma solución optimiza al mismo tiempo tanto la función objetivo clásica como la función objetivo minisuma. Lo mismo ocurre para la función minimax, que en los casos mencionados también se encuentra en una misma región de indiferencia que la función objetivo clásica.

Para las DMUs Abaeté y ABSA las soluciones que optimizan la función objetivo referente al modelo DEA-BCC recubren todo el espacio de los pesos. Así, según el análisis realizado en J.C.N. Clímaco, J.C.C.B. Soares de Mello y L. Angulo-Meza (2008) estas deberán ser las DMUs con la mejor evaluación en el modelo MCDEA-BCC.

Las DMUs TAM y el Grupo Varig son minisuma eficientes, pero no minimax eficientes. Sin embargo, la DMU TAM y el Grupo Varig dejaron de ser minimax eficientes apenas por un área muy pequeña $(0,02 \%$ del área del espacio de los pesos). Así en análisis cualitativo la TAM debe ser mejor evaluado que el Grupo Varig. Por otro lado, la GOL fue apenas eficiente y, aún así, en un área muy pequeña, solo consiga la eficiencia con conjuntos muy particulares de multiplicadores.

La Tabla 2 muestra el índice de eficiencia MCDEATRIMAP, a través de la suma ponderada de las soluciones de la primera función objetivo, cuyos pesos son los porcentajes de las áreas en que cada solución es válida. Los dados son todos proporcionados por el TRIMAP.

Tabla 2. Índice MCDEA-TRIMAP.

\begin{tabular}{|c|c|}
\hline $\begin{array}{c}\text { Compañías aéreas } \\
\text { brasileñas en 2005 }\end{array}$ & $\begin{array}{c}\text { Índice } \\
\text { MCDEA-TRIMAP }\end{array}$ \\
\hline Abaeté Linhas Aéreas & 1,000000 \\
\hline Absa - Aerolineas Brasileiras & 1,000000 \\
\hline Tam Linhas Aéreas & 0,999996 \\
\hline Grupo Varig & 0,999390 \\
\hline Gol Transportes Aéreos & 0,952333 \\
\hline Meta Mesquita & 0,613453 \\
\hline Passaredo Transportes Aéreos & 0,494743 \\
\hline Puma Air Linhas Aéreas & 0,490256 \\
\hline Trip Linhas Aéreas & 0,455677 \\
\hline Rico Linhas Aéreas & 0,420171 \\
\hline Pantanal Linhas Aéreas & 0,212925 \\
\hline Taf Linhas Aérea & 0,196084 \\
\hline Total Linhas Aéreas & 0,194015 \\
\hline Oceanair & 0,118447 \\
\hline
\end{tabular}

La evaluación de las compañías aéreas a través del modelo MCDEA-BCC no genera metas. Para la determinación de las compañías aéreas con los mejores desempeños en el año 2005 es importante segmentarlas en clusters, capaces de agrupar DMUs con características gerenciales semejantes. Así, el proceso de determinación de benchmarks se vuelve más eficaz, al apuntar las metas en un grupo de empresas con el mismo tipo de operación. Para 
la determinación de los clusters, se realizó una comparación entre los multiplicadores atribuidos por el modelo a cada variable de cada DMU. Para el cálculo de los multiplicadores se utilizó el software LINDO 6.1.

Tal y como se ha mencionado, los valores encontrados se utilizaron para sementar las DMUs, en primer lugar con relación a la gestión de sus recursos (inputs). Así se generaron dos clusters para todas las compañías. El cluster 1 se caracteriza por las compañías aéreas que atribuyeron un multiplicador mayor para el input peso máximo de decolaje que para el input personal. Esas empresas tienen la capacidad como recurso más valorizado en la evolución del desempeño.

El cluster 2 está formado por las empresas que atribuyeron un multiplicador mayor para la variable personal que para la variable capacidad (peso máximo de decolaje). En este caso, las compañías dan mayor valor al input personal, siendo este recurso el principal responsable por la evaluación de la empresa en este año.

De esta misma forma, se definieron dos clusters a partir de los multiplicadores atribuidos por cada empresa a sus outputs. Estos clusters definen el principal foco operacional de las empresas, ya que indican que output influenció más en su desempeño.
El cluster 3 está formado por las compañías aéreas que tuvieron un multiplicador mayor para el output asientos por kilómetros utilizados que para el output toneladas por kilómetros utilizados. En este caso, las compañías aéreas consideran como su negocio principal el transporte de pasajeros y no el de carga.

El cluster 4 está compuesto por las empresas que tiene el transporte de carga como su principal negocio. Las compañías de este cluster dieron un multiplicador mayor para el output toneladas por kilómetros utilizados que para el output asientos por kilómetros utilizados.

Los clusters definidos para las compañías aéreas están descritos en la Figura 6, donde se encuentran destacados los benchmarks (como la compañía que obtuvo el mayor índice MCDEA-TRIMAP).

\section{CONCLUSIONES}

El índice MCDEA-TRIMAP viabilizó la realización de un análisis más completo del modelo en estudio, con lo que se identificó a la ABSA como realmente eficiente en su ramo de actuación, que es el transporte de cargas y que actúa básicamente en el norte del Brasil y en algunas ciudades de América Latina. La compañía Abaeté es una compañía regional, que opera en apenas tres ciudades del estado brasileño de Bahia. Como esta compañía es pequeña, ella se

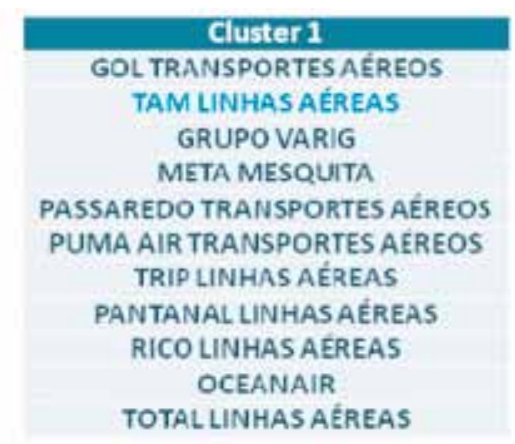

Cluster2

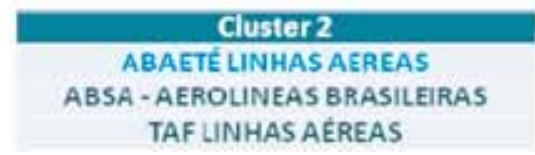

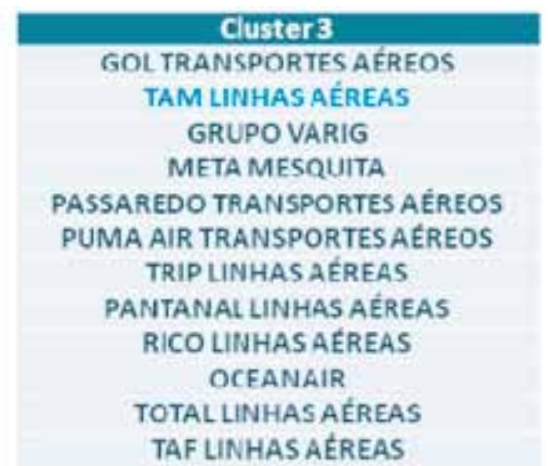

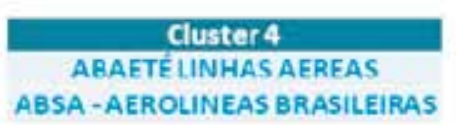

Figura 6. Clusters de las Compañías Aéreas en 2005. 
benefició de los retornos variables de escala del modelo BCC, siendo eficiente por default.

El Grupo Varig fue casi $100 \%$ eficiente. El resultado mostrado es coherente, aunque esta compañía estuviese en estado de previo a la quiebra en el año 2005 y efectivamente, entraría en colapso en 2006. Esto fue debido a que en el año 2005, el Grupo Varig pasó por un choque de gestión con el objetivo a finalmente aumentar el uso de la flota, reducir empleado y costos operacionales. Por lo tanto, los resultados obtenidos muestran el éxito de este esfuerzo en términos de su eficiencia operacional. Los resultados aquí mostrados no consideran variables monetarias, ni la acumulación de los problemas anteriores, como el code-share con la TAM [27].

La segmentación del mercado de transporte aéreo permite identificar a la TAM como benchmarks de los clusters 1 y 3 , pues esta mostró el mejor índice de eficiencia MCDEA-TRIMAP con relación a las demás. Esto se puede justificar por el crecimiento de esta compañía a lo largo de los años anteriores, previamente detectado, con el aumento de rutas y vuelos.

Para el cluster 2, el benchmark para la TAF es la Abaeté, pues ambas transportan tanto carga como pasajeros. El cluster 4 está compuesto por la compañía ABSA, una empresa exclusivamente carguera, justificando su clasificación en este cluster compuesto por empresas que tienen un foco mayor en el transporte de cargas. Por otro lado, la Abaeté Linhas Aéras también transporta pasajeros, sin embargo el índice de eficiencia calculado tiene mayor influencia del transporte de cargas.

Se puede notar un grande potencial de este estudio en lo que se refiere al desempeño operacional en el transporte aéreo, pues no existen muchos estudios con este enfoque. Gran parte de las evaluaciones de las compañías aéreas están basados en los resultados económico-financieros de estas empresas, sin tener en cuenta los aspectos operacionales que generan estos resultados.

El modelo MCDEA [20] se desarrolló para modelos DEA-CCR. Este estudio también contribuye con la adaptación del modelo para el BCC, lo que posibilita una mejor evaluación, pues el mercado de transporte aéreo puede mostrar retornos de escala variables. Estudios futuros podrían explotar el mejor desarrollo del modelo MCDEA para el modelo DEA-BCC con la utilización de la herramienta TRIMAP. Esto se debe a que estas herramientas muestra ciertas inestabilidades numéricas, que algunas veces imposibilitan el análisis.

\section{AGRADECIMIENTOS}

Agradecemos el apoyo financiero del $\mathrm{CNPq}$ (Conselho Nacional de Desenvolvimento Científico e Tecnologico), Brasilia, y de la FAPERJ (Fundação Carlos Chagas Filho de Amparo à Pesquisa do Estado do Rio de Janeiro), Rio de Janeiro, instituciones de fomento del Brasil.

\section{REFERENCIAS}

[1] A.V.M. Oliveira. "A experiência brasileira na desregulamentação do transporte aéreo: um balanço e propositura de diretrizes para novas políticas". SEAE/MF. Documento de Trabalho $N^{\circ}$ 5. 2007.

[2] R. Coelho. "A Construção da agenda de desregulamentação do setor de transporte aéreo no Brasil". In XVI ANPET, Natal, pp. 383-394. 2002.

[3] J.C.N. Clímaco and C.H. Antunes. "TRIMAPan interactive tricriteria linear programming package". Foundations of Control Engineering. Vol. 12, pp. 101-119. 1987.

[4] J.C.C.B. Soares de Mello, J.C.N. Clímaco y L. Angulo-Meza. "Índice de eficiência MCDEA-TRIMAP”. In XXXVIII Simposio Brasileiro de Pesquisa Operacional Goiania. 2006.

[5] A.H. Araújo, J.V.G. Avellar, A.Z. Millioni e F.A.S. Marins. "Eficiência e Desempenho do Transporte Aéreo Regional Brasileiro". In Simpósio de Pesquisa Operacional e Logística da Marinha, pp. 1-10. Rio de Janeiro, Brasil. 2006.

[6] J.C.C.B. Soares de Mello, L. Angulo-Meza, E.G. Gomes, B.P. Serapião e M.P.E. Lins. "Análise de envoltória de dados no estudo da eficiência e dos benchmarks para companhias aéreas brasileiras". Pesquisa Operacional. Vol. 23, pp. 325-345. 2003. 
[7] E. Fernandes and H.M.P. Capobianco. "Airline capital structure and returns". Journal of Air Transport Management. Vol. 7, pp. 137-142. 2001.

[8] H.M.P. Capobianco and E. Fernandes. "Capital structure in the world airline industry". Transportation Research Part a-Policy and Practice. Vol. 38, pp. 421-434. 2004.

[9] J.Q. Silveira, E.R. Pereira, T.C.V.D. Correia, J.C.C.B. Soares de Mello, J.C.N. Climaco e L. Angulo-Meza. "Avaliação da eficiência das companhias aéreas brasileiras com uma variação do modelo de Li e Reeves". Engevista. Vol. 10, pp. 145-155. 2008.

[10] E. Fernandes and R.R. Pacheco. "Efficient use of airport capacity". Transportation Research Part a-Policy and Practice. Vol. 36, pp. 225-238. 2002.

[11] R.R. Pacheco and E. Fernandes. "Managerial efficiency of Brazilian airports". Transportation Research Part a-Policy and Practice. Vol. 37, pp. 667-680. 2003.

[12] R.R. Pacheco, E. Fernandes and M.P.D. Santos. "Management style and airport performance in Brazil". Journal of Air Transport Management. Vol. 12, pp. 324-330. 2006.

[13] J.C.C.B. Soares de Mello e E.G. Gomes. "Eficiências aeroportuárias: uma abordagem comparativa com análise de envoltória de dados". Revista de Economia e Administração. Vol. 3, pp. 15-23. 2004.

[14] A. Charnes, W.W. Cooper and E. Rhodes. "Measuring the efficiency of decision-making units". European Journal of Operational Research. Vol. 2, pp. 429-444. 1978.

[15] R.D. Banker, A. Charnes and W.W. Cooper. "Some models for estimating technical scale inefficiencies in data envelopment analysis". Management Science Vol. 30, pp. 1078-1092. 1984.

[16] F.R. Leta, J.C.C.B. Soares de Mello, E.G. Gomes e L. Angulo-Meza. "Métodos de melhora de ordenação em DEA aplicados à avaliação estática de tornos mecânicos". Investigação Operacional. Vol. 25, pp. 229242. 2005.

[17] T.R. Sexton, R.H. Silkman and A.J. Logan. "Data Envelopment Analysis: Critique and extensions". En Measuring efficiency: An assessment of data envelopment analysis. Jossey-Bass Editor. Edição ed. San Francisco, pp. 73-105. 1986.

[18] B. Golany and Y. Roll. "An application procedure for DEA". Omega. Vol. 17, pp. 237-250. 1989.

[19] L. Angulo-Meza and M.P.E. Lins. "Review of methods for increasing discrimination in data envelopment analysis". Annals of Operations Research. Vol. 116, pp. 225-242. 2002.

[20] X.-B. Li and G.R. Reeves. "A multiple criteria approach to data envelopment analysis". European Journal of Operational Research. Vol. 115, pp. 507-517. 1999.

[21] J.C.C.B. Soares de Mello, M.P.E. Lins and E.G. Gomes. "Construction of a smoothed DEA frontier". Pesquisa Operacional. Vol. 28, pp. 183-201. 2002.

[22] J.C.N. Climaco and C.H. Antunes. "Implementation of a user-friendly software package. A guided tour of TRIMAP". Mathematical and Computer Modelling. Vol. 12, pp. 1299-1309. 1989.

[23] J.C.N. Climaco, C.H. Antunes e M.J. Alves. "Programação Linear Multiobjectivo". Imprensa da Universidade de Coimbra. Edição ed. Coimbra. Portugal. 2003.

[24] T.C.V.D. Correia e J.C.C.B. Soares de Mello. "Avaliação da eficiência das companhias aéreas brasileiras com modelo DEA nebuloso". In XII Congresso de Pesquisa e Ensino em Transportes-ANPET. Panorama Nacional da Pesquisa em Transportes 2008 Fortaleza: ANPET, pp. 975-985. 2008.

[25] L. Angulo-Meza, L. Biondi Neto, J.C.C.B. Soares de Mello and E.G. Gomes. "ISYDS - Integrated System for Decision Support (SIAD Sistema Integrado de Apoio a Decisão): A Software Package for Data Envelopment Analysis Model". Pesquisa Operacional. Vol. 25, pp. 493-503. 2005.

[26] J.C.N. Clímaco, J.C.C.B. Soares de Mello and L. Angulo-Meza. "Performance Measurement -From DEA to MOLP”. En Encyclopedia of Decision Making and Decision Support Technologies. Information Science Reference. Edição ed. Hershey. Vol. 2, No 2, pp. 709715. 2008. 
[27] P.H.C. Soares de Mello, J.C.C.B. Soares de Mello and L. Angulo-Meza. "Misunderstandings due to a codeshare between two Brazilian airlines in Rio de Janeiro international airport". Rio's International Journal on sciences of industrial and systems engineering and management. Vol. 3, p. 2. 2009. 